\title{
Supplemental oxygen did not reduce progression of pre-threshold retinopathy of prematurity in infants
}

The STOP-ROP Multicenter Study Group. Supplemental therapeutic oxygen for prethreshold retinopathy of prematurity (STOP-ROP), a randomized, controlled trial. I: primary outcomes. Pediatrics 2000 Feb;105:295-310.

QUESTION: In premature infants, is supplemental oxygen at pulse oximetry saturations of $96 \%$ to $99 \%$ more effective than conventional oxygen at pulse oximetry saturations of $89 \%$ to $94 \%$ in reducing the progression from moderate (pre-threshold) to threshold retinopathy of prematurity $(\mathrm{ROP})$ ?

Design

Randomised (allocation concealed), blinded (outcome assessor), controlled trial with 3 months of follow up.

\section{Setting}

71 hospitals affiliated with 30 centres in the US.

\section{Patients}

649 infants (mean gestational age 25 wks, $57 \%$ boys, $55 \%$ white) who were born prematurely, had prethreshold ROP in $\geqslant 1$ eye, and had a median pulse oximetry $\leq 94 \%$ oxygen saturation in room air. Infants were excluded if they had lethal anomalies or congenital anomalies of the eye. Follow up was $92 \%$.

\section{Intervention}

After stratification by study centre and 2 levels of baseline ROP severity, infants were randomised to receive supplemental oxygen therapy with pulse oximetry targeted at $96 \%$ to $99 \%$ oxygen saturation (supplemental group, $\mathrm{n}=324$ ) or conventional oxygen therapy with pulse oximetry targeted at $89 \%$ to $94 \%$ oxygen saturation (conventional group, $\mathrm{n}=325$ ) for $\geqslant 2$ weeks.

\section{Main outcome measures}

The main outcome measure was the rate of progression to threshold ROP in $\geqslant 1$ eye. Secondary outcome measures were other ophthalmic and adverse events. Infants had weekly eye examinations by an ophthalmologist.

Sources of funding: National Institute of Child Health and

Human Development; National Institute of Nursing Research;

National Institutes of Health;Delta Gamma Sorority; Rhe and Raymond White Donation; Research to

Prevent Blindness, Chartwell Home Therapy; Ohmeda Corporation.

For correspondence: Dr D L Phelps,

Children's Hospital at Strong, University of Rochester School of

Medicine and Dentistry,

Pediatrics, Box 651,601

Elmwood Avenue,

Rochester, NY 14642,

USA.Fax +1 716461

3614.

\section{Main results}

Analysis was by intention to treat. Infants in the conventional and supplemental groups did not differ for rates of progression to threshold ROP in $\geqslant 1$ eye ( $48 \% v 41 \%)$ $\{\mathrm{p}=0.06\}^{*}$, retinal detachments or folds $(4.4 \% v 4.1 \%)$, or macular ectopia $(3.9 \%$ v 3.9\%). Compared with infants in the conventional group, more infants in the supplemental group remained in hospital ( $7 \%$ v 13\%, $\mathrm{p}=0.01)$, remained on oxygen $(37 \% v 47 \%, \mathrm{p}=0.02)$, remained on diuretics (24\% v 36\%, $\mathrm{p}=0.002)$, and had $\geqslant 1$ adverse pulmonary event defined as remaining in oids, methylxanthines, or diuretics $(46 \%$ v $57 \%$, $\mathrm{p}=0.005)$.

\section{Conclusion}

Supplemental therapeutic oxygen at pulse oximetry saturations of $96 \%$ to $99 \%$ did not decrease the rate of progression from pre-threshold to threshold retinhospital or remaining on study equipment, oxygen, ster- opathy of prematurity in infants but increased the risk of adverse pulmonary events.

*p Value calculated from data in article.

\section{COMMENTARY}

The aetiology and course of ROP has long been an issue of debate in neonatal circles. Preterm infants who develop ROP appear to have lower birth weights, a more complex medical course, and a longer duration of oxygen administration. ${ }^{1}$ Thus, the aetiology appears to be multifactorial, making cause and effect difficult to establish. Supplemental oxygen to decrease hypoxic stimulus in the retina is a biologically sound assumption. This multicentre, well controlled, randomised study, however, did not support this premise.

Study strengths include use of many sites (30 US centres), randomisation to supplemental or conventional group, a power level of $80 \%$ to detect differences between groups $(\mathrm{n}=649)$, and $92 \%$ follow up. In the study, supplemental oxygen was defined as oxygen needed to maintain saturations at $96 \%$ to $99 \%$ for most infants. This was an approximate $10 \%$ increase in $\mathrm{FIO}_{2}$ over the control group, which had saturations maintained at $89 \%$ to $94 \%$. Infants who were in the supplemental oxygen group were 1.8 times more likely to develop adverse pulmonary events such as pneumonia or cardiovascular disease than those in the control group.

The study findings are important for healthcare providers working with preterm infants with ROP because of the worry about adequate saturation levels to support the retina. However, given the potential increased risks of chronic lung disease, respiratory related needs should be a focus of concern in these medically complex infants. Using supplemental oxygen does not appear to be advantageous for these infants. This study, however, examined oxygen supplementation after diagnosis of pre-threshold ROP and therefore cannot be used to make assumptions about oxygen supplementation before this time. Lastly, a subgroup analysis found that infants with pre-threshold ROP without plus disease (dilated and tortuous vessels in $\geq 2$ quadrants of the posterior pole) who received supplemental oxygen appeared to benefit from the treatment. More research is needed to confirm these results.

Jacqueline M McGrath, RN, NNP, CGNS, PhD Assistant Professor, College of Nursing Arizona State University Tempe, Arizona, USA

1 Phelps DL. Retinopathy of prematurity. Pediatr Rev 1995;16:50-6. 